\title{
Emergent Sleeve Gastrectomy for Gastric Necrosis Resulting From Lap Band Slippage
}

\author{
Minal Joshi, MD, Krystyna Kabata, PA-C, Srikanth Earhiraju, MD, Michael Baek, MD, \\ Piotr J. Gorecki, MD \\ Department of Surgery, New York Methodist Hospital, Brooklyn, NY, USA (all authors).
}

\begin{abstract}
Introduction: Laparoscopic adjustable gastric banding (LAGB) has a potential for long-term complications. We report a case of LAGB slippage with extensive gastric necrosis managed with emergent sleeve gastrectomy.

Case Report: A 45-year-old man presented to the emergency department after returning from a distant trip and reported a 3-day history of progressively severe abdominal pain, nausea, vomiting, and fever. He had undergone placement of the LAGB 2 years before this presentation, which resulted in subsequent weight loss of $143 \mathrm{lb}$ and resolution of his comorbidities. On admission, the patient was hypotensive, tachycardic, and oliguric, with evident peritonitis. A computed tomography scan revealed extensive intraperitoneal free air and intra-abdominal fluid. After intravenous fluid resuscitation, he underwent emergent exploratory laparoscopy. A slipped band with gastric prolapse and extensive gastric necrosis were found, with multiple perforations involving most of the greater curvature of the stomach. The LAGB was explanted and a laparoscopic sleeve gastrectomy was performed. A liquid diet was introduced on postoperative day 4 . Immediate recovery was prolonged because of acute-onset chronic renal failure and requirement for optimization of nutrition. The patient was discharged home on postoperative day 13 and had a subsequent uneventful recovery.
\end{abstract}

Conclusion: Gastric prolapse complicated by gastric necrosis is a rare life-threatening complication of LAGB. Once acute LAGB slippage is suspected, urgent attention and treatment are needed to minimize the chance of gastric ischemia. Laparoscopic explanation of LAGB and emergent sleeve gastrectomy may be considered in similar clinical settings to optimize the outcome and minimize the morbidity of near total or total gastrectomy.

Key Words: Laparoscopic adjustable gastric banding, Morbid obesity, Gastric necrosis, Sleeve gastrectomy, Gastric prolapse, Lap band slip.

Citation Joshi M, Kabata K, Earhiraju S, Baek M, Gorecki PJ. Emergent sleeve gastrectomy for gastric necrosis resulting from lap band slippage. CRSLS e2014.00180. DOI: $10.4293 /$ CRSLS.2014.00180.

Copyright (C) 2014 SLS This is an open-access article distributed under the terms of the Creative Commons Attribution-Noncommercial-ShareAlike 3.0 Unported license, which permits unrestricted noncommercial use, distribution, and reproduction in any medium, provided the original author and source are credited.

Presented at the 2013 Annual SAGES Meeting, April 17-20, 2013, Baltimore, Maryland.

Address correspondence to: Minal Joshi, MD, New York Methodist Hospital, Department of Surgery, 506 Sixth Street, Brooklyn, NY 11215. Telephone: (718) 780-3279, Fax: (718) 780-3154, E-mail: minuday2000@gmail.com

\section{INTRODUCTION}

More than one-third of adults in the United States are considered obese. ${ }^{1}$ Since its introduction in 1993, laparoscopic adjustable gastric banding (LAGB) has gained popularity as the least invasive surgical treatment for morbid obesity. ${ }^{2}$ However, with its recognized shortterm advantages, it also carries a risk of potential longterm complications. We report an unusual case of band "slippage," with extensive gastric necrosis treated with emergent laparoscopic sleeve gastrectomy.

\section{CASE REPORT}

A 45-year-old man presented to the emergency department after returning from a distant trip and reported a 3-day history of progressively severe abdominal pain, nausea, vomiting, and fever. He had undergone laparoscopic placement of an LAGB (initial weight, $344 \mathrm{lb}$; body mass index, $50 \mathrm{~kg} / \mathrm{m}^{2}$ ) 24 months before this presentation. The pars flaccida technique and 2-suture gastric plication were used, with a subsequent uneventful and prompt recovery. The patient had a subsequent weight loss of 143 


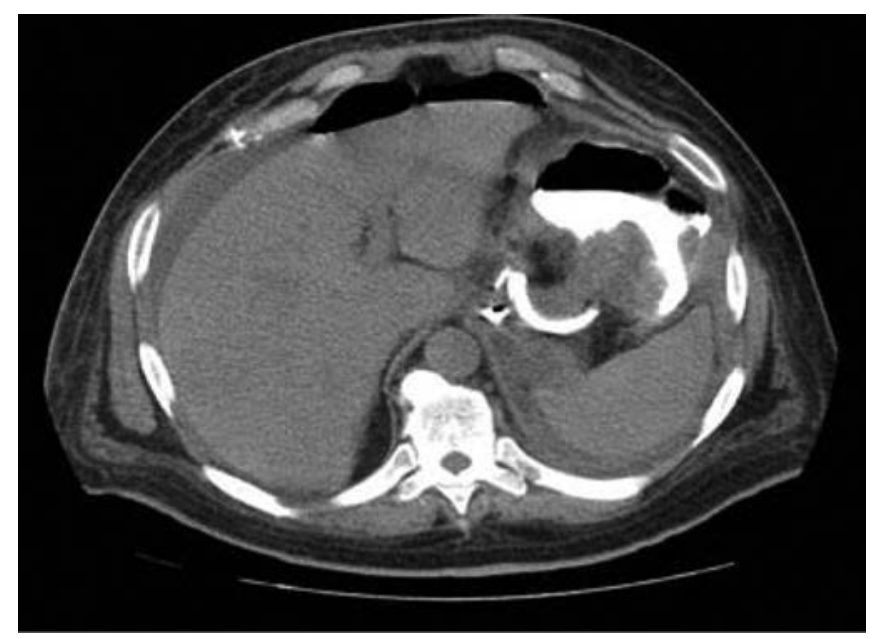

Figure 1. Computed tomography scan of the abdomen showing free air, free fluid, and the slipped band.

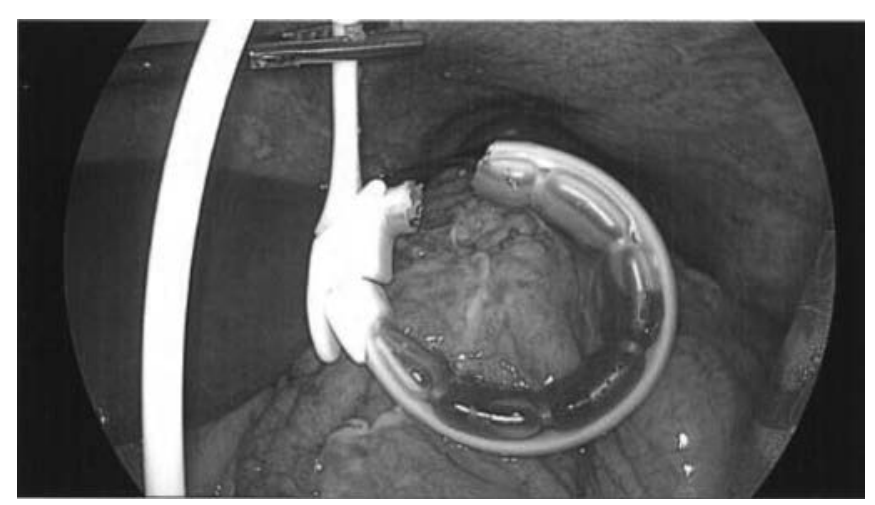

Figure 2. Laparoscopic view showing the explanted gastric band and peritonitis.

$\mathrm{lb}$ and improvement in his comorbidities, including type 2 diabetes, hypertension, hyperlipidemia, and blindness, and he reported having an excellent quality of life. On the day of presentation to the emergency department, his weight was $201 \mathrm{lb}$ with a body mass index of $30.7 \mathrm{~kg} / \mathrm{m}^{2}$. He was hypotensive, tachycardic, and oliguric. Physical examination revealed signs of peritonitis. Laboratory results showed elevated serum glucose, blood urea nitrogen, and creatinine levels. His arterial blood gas results were $\mathrm{pH}$ 7.41, $\mathrm{pCO}_{2}, 33 \mathrm{mg} \mathrm{Hg} ; \mathrm{pO}_{2}, 67 \mathrm{~mm} \mathrm{Hg}$; and $\mathrm{HCO}_{3}, 20.6 \mathrm{mmol} / \mathrm{L}$. A nasogastric tube was inserted, and an urgent noncontrast computed tomography scan of the abdomen and pelvis was done to delineate the positioning of the lap band in the face of diffuse peritonitis. A noncontrast abdominal CT scan (Figure 1) revealed extensive free air and free intra-abdominal fluid, with edematous changes in the mesentery. The patient was given fluid resuscitation of $4 \mathrm{~L}$ of normal saline, followed by emergent exploratory laparoscopic surgery. At laparoscopy, a "slipped" gastric band was found, along with extensive gastric necrosis and multiple perforations involving most of the greater curvature of the stomach and fundus, which was prolapsed via the band (Figures 2 and 3). There was evident diffuse peritonitis. The LAGB was explanted, and a laparoscopic sleeve gastrectomy of the gangrenous and ischemic parts of the stomach was performed. At the level of the incisura, gastric ischemia was found to extend to the lesser curvature (Figure 4), with no necrosis as confirmed by intraoperative upper endoscopy. Although endoscopy may not be the most reliable method to evaluate for gastric perfusion, the absence of necrosis in the resected margin may allow the performance of the less extensive gastrectomy in this critical setting in a concept of the damage control procedure. As a result, the decision was made to preserve this segment as a "sleeve" and avoid the need for a near total gastrectomy with a Roux-en-Y gastrojejunostomy reconstruction, which would have been a very high-risk procedure in this hypotensive, septic, and malnourished patient. In the similar setting, the creation of the gastric sleeve was more conservative and dictated by the local anatomic and physiologic factors rather than by the need for creation of the optimal gastric restriction. The principles of a damage control procedure and conservative resection constituted the priority over the long-term restriction and resultant weight loss. Pathologic examination of the resected stomach confirmed extensive transmural necrosis with multiple perforations. Parenteral nutrition was started on postoperative day 1 and a liquid diet begun on postoperative day 4. Postoperative recovery was prolonged because of acute-onset chronic renal failure and the need for nutritional support. On the day

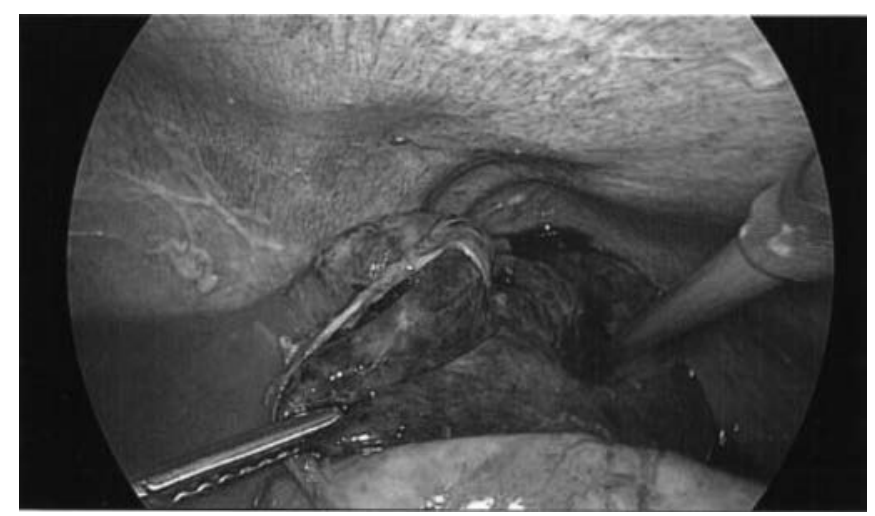

Figure 3. Laparoscopic view of the greater curvature of the stomach. Extensive and advanced necrosis with multiple perforations can be seen. 


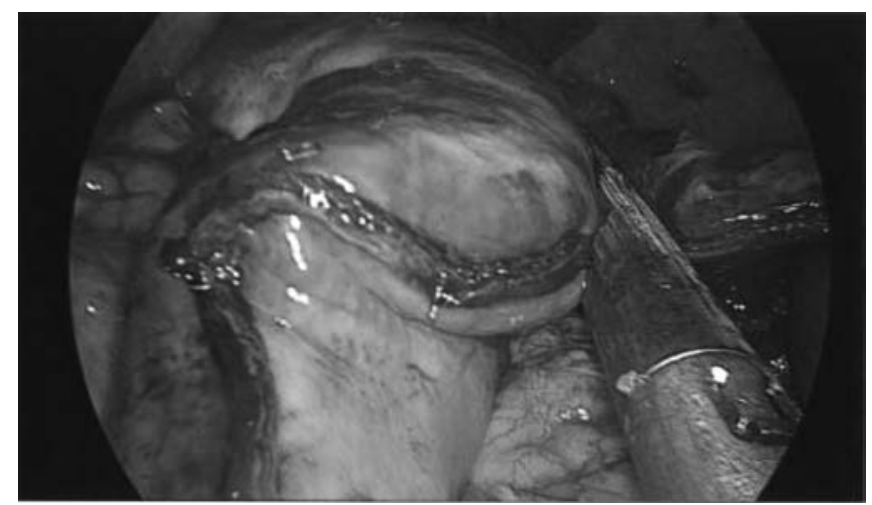

Figure 4. Laparoscopic view of the sleeve gastrectomy of the greater curvature. The lesser curvature and "sleeve gastrectomy" were left in situ.

of admission, the patient's serum albumin level was $2.6 \mathrm{~g} / \mathrm{mL}$ and his prealbumin was at nadir on postoperative day 3 at the level of $5 \mathrm{mg} / \mathrm{dL}$. On the day of discharge, his albumin level improved to $3.1 \mathrm{~g} / \mathrm{dL}$ and his prealbumin level to 17 $\mathrm{mg} / \mathrm{dL}$. He was discharged home on postoperative day 13, and his further recovery was uneventful.

\section{DISCUSSION}

LAGB is considered an established bariatric operation favored by many patients and surgeons because of its simplicity, minimal invasiveness, and reversibility. ${ }^{3}$ However, LAGB does carry a chance of a long-term morbidity. The long-term follow-up data incorporating contrast radiograms and endoscopic examinations indicate a number of potential significant late complications. The common causes for reoperation include band slippage, erosion, pouch dilatation, leakage from the lap band system, and esophageal dilatation. ${ }^{4}$ One of the most severe complications of LAGB may be gastric ischemia with perforation. ${ }^{5}$ The gastric necrosis caused by band slippage or gastric prolapse requires immediate recognition and urgent surgical intervention if gastric ischemia is suspected. ${ }^{6}$

Table 1 presents the characteristics of 10 patients with gastric necrosis after LAGB, reported in the literature over the past 10 years. All patients summarized in the table, similar to our presented case, had acute symptoms of upper gastrointestinal obstruction at the time of their presentation. All patients underwent urgent surgery, and the sleeve gastrectomy was the most frequently performed

Table 1.

Literature Review: Patients With Gastric Necrosis Induced by Gastric Banding

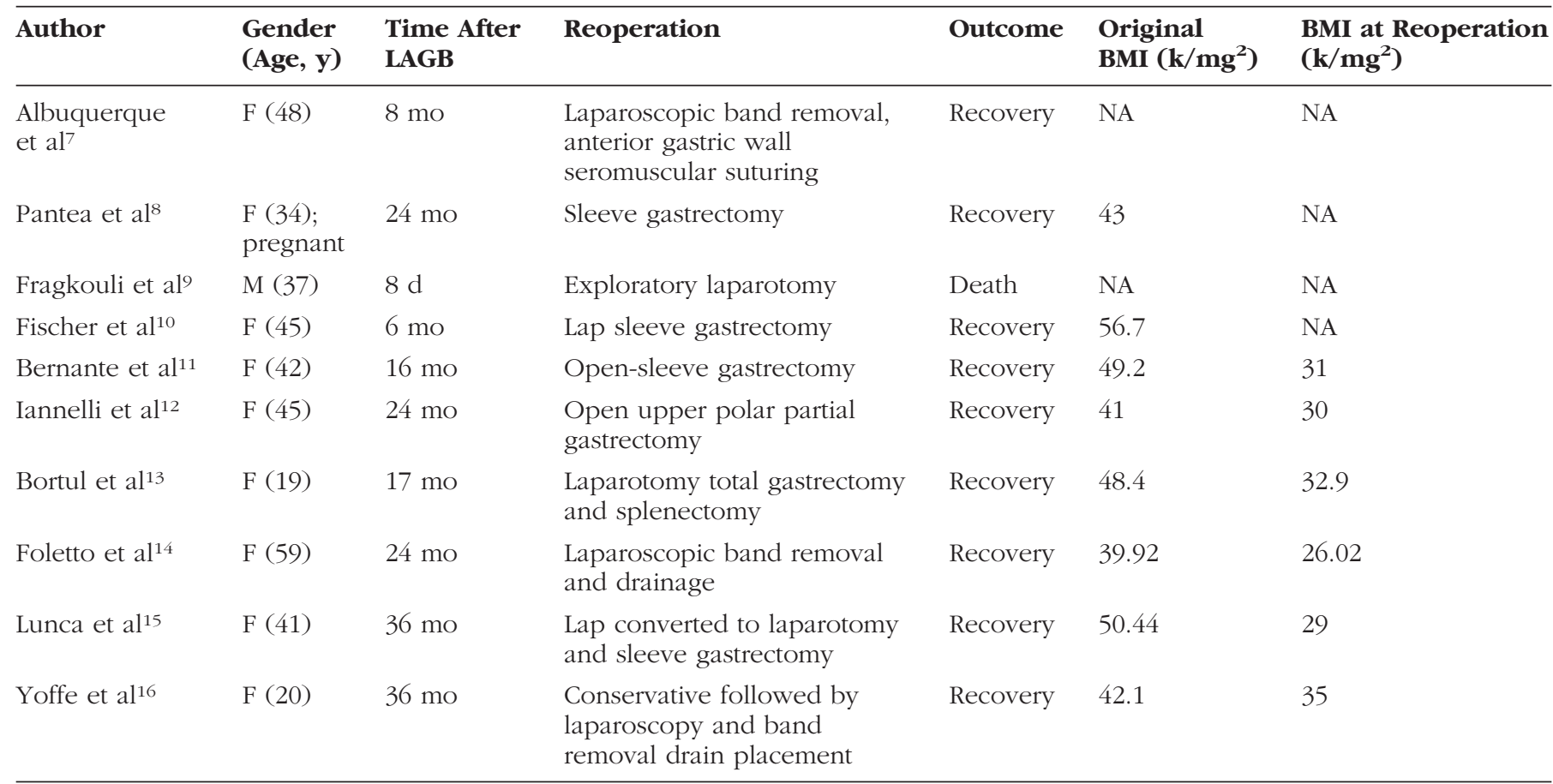

NA, not available. 
operation. One patient was treated with laparoscopic intervention including band removal and seromuscular suturing, while another patient died despite prompt exploratory laparotomy. In the presented case, gastrectomy was unavoidable because of extensive gastric necrosis and multiple perforations. A viable lesser curvature allowed for the performance of emergent sleeve gastrectomy within the margins of viable tissue.

Although the complication of band slippage with gastric necrosis is rare, once ischemia is suspected, it is essential to recognize and treat the patient immediately to avoid mortality. In the presence of adequate viable gastric cardia and lesser curvature, laparoscopic explantation of an LAGB and emergent sleeve gastrectomy should be considered an appropriate procedure that may lower the morbidity associated with the alternatives of emergent subtotal gastrectomy (no viable distal stomach) or a Roux-en-Y gastric bypass (viable remnant distal stomach). In a rare situation, if there is no viable gastric cardia, total gastrectomy with esophagojejunostomy would be required.

\section{References:}

1. Ogden CL, Carroll MD, Kit BK, et al. Prevalence of obesity in the United States, 2009-2010. NCHS data brief, No. 82. Hyattsville, MD: National Center for Health Statistics; 2012.

2. Mehanna MJ, Birjawi G, Moukaddam HA, et al. Complications of adjustable gastric banding, a radiological pictorial review. AJR Am J Roentgenol. 2006;186:522-534.

3. Provost DA. Laparoscopic adjustable gastric banding: an attractive option. Surg Clin North Am. 2005;85:789-805.

4. Gustavsson S, Westling A. Laparoscopic adjustable gastric banding: complications and side effects responsible for the poor long-term outcome. Semin Laparosc Surg. 2002;9(2):115-124.

5. Chelala E, Cadiére GB, Favretti F, et al. Conversions and complications in 185 laparoscopic adjustable silicone gastric banding cases. Surg Endosc. 1997;11(3):268-271.
6. Spivak H, Favretti F. Avoiding postoperative complications with the LAP-BAND system. Am J Surg. 2002;184:208-218.

7. Albuquerque A, Bastos P, Macedo G. Gastric necrosis caused by gastric banding. Gastrointest Endosc. 2012;75(5):1098, discussion 1098-1099.

8. Pantea S, Pantea C, Duţă C, et al. Gastric necrosis, rare complication after laparoscopic gastric banding-case report. Chirurgia (Bucur). 2011;106(1):109-112.

9. Fragkouli K, Mitselou A, Vougiouklakis T. Death-related gastric necrosis after laparoscopic adjustable gastric banding in the early post-operative period. Diagn Pathol. 2010;5:68.

10. Fischer G, Myers JA, Huang W, Shayani V. Gastric migration and strangulation after adjustable gastric banding. Obes Surg. 2008;18(6):753-775.

11. Bernante P, Breda C, Zangrandi F, Pomerri F, Pelizzo MR, Foletto M. Emergency sleeve gastrectomy as rescue treatment for acute gastric necrosis due to type II paraesophageal hernia in an obese woman with gastric banding. Obes Surg. 2008;18(6):737741 .

12. Iannelli A, Facchiano E, Sejor E, Baqué P, Piche T, Gugenheim J. Gastric necrosis: a rare complication of gastric banding. Obes Surg. 2005;15(8):1211-1214.

13. Bortul M, Scaramucci M, Tonello C, Spivach A, Liguori G. Gastric wall necrosis from organo-axial volvulus as a late complication of laparoscopic gastric banding. Obes Surg. 2004;14(2): $285-287$

14. Foletto M, De Marchi F, Bernante P, Busetto L, Pomerri F. Late gastric pouch necrosis after Lap-Band, treated by an individualized conservative approach. Obes Surg. 2005;15(10):14871490.

15. Lunca S, Vix M, Rikkers A, Rubino F, Marescaux J. Late gastric prolapse with pouch necrosis after laparoscopic adjustable gastric banding. Obes Surg. 2005;15(4):571-575.

16. Yoffe B, Sapojnikov S, Goldblum C. Gastric wall necrosis following late prolapse after laparoscopic banding. Obes Surg. 2004;14(1):142-144. 